\title{
Detection of human telomerase reverse transcriptase mRNA in cells obtained by lavage of the pleura is not associated with worse outcome in patients with stage I/II non-small cell lung cancer: Results from Cancer and Leukemia Group B 159902
}

\author{
Alice M. Boylan, MD, ${ }^{\mathrm{a}}$ Xiaofei F. Wang, PhD, ${ }^{\mathrm{b}}$ Richard Ko, DO, ${ }^{\mathrm{a}}$ Patricia M. Watson, $\mathrm{PhD},{ }^{\mathrm{a}}$ Lin Gu, MS, \\ David Harpole, MD ${ }^{\mathrm{c}}$ Raphael Bueno, MD, ${ }^{\mathrm{d}}$ Rosemary Kelly, MD, ${ }^{\mathrm{e}}$ Leslie Kohman, MD, ${ }^{\mathrm{f}}$ and \\ Robert Kratzke, $\mathrm{MD}^{\mathrm{g}}$
}

\begin{abstract}
Objective: Previous studies suggest that cytologic analysis of cells obtained by lavage of the pleural surfaces at the time of resection of non-small cell lung cancer can identify patients at risk for recurrence. Because telomerase gene expression has been associated with worse outcome in non-small cell lung cancer, we hypothesized that identification of cells obtained from pleural lavage that express telomerase would identify patients at risk for recurrent disease.
\end{abstract}

\begin{abstract}
Methods: Patients with presumed non-small cell lung cancer underwent thoracotomy with curative intent. Cells obtained by lavage of the pleural surfaces were analyzed for telomerase catalytic subunit human telomerase reverse transcriptase mRNA expression using reverse transcriptase polymerase chain reaction.
\end{abstract}

\begin{abstract}
Results: A total of 194 patients with stage I/II non-small cell lung cancer had adequate samples, and median follow-up was 60 months (17-91 months). By using Cox models, no statistical differences were found between human telomerase reverse transcriptase-negative and positive patients in disease-free survival (hazard ratio, 1.28 ; $95 \%$ confidence interval, $0.85-1.94$; log-rank test, $P=.2349$ ) or overall survival (hazard ratio, 1.13; $95 \%$ confidence interval, $0.72-1.79$; log-rank test, $P=.5912$ )

Conclusions: Detection of human telomerase reverse transcriptase in cells obtained from pleural lavage of patients with stage I/II non-small cell lung cancer does not identify patients at risk for recurrent disease. (J Thorac Cardiovasc Surg 2013;146:206-11)
\end{abstract}

Local recurrences after surgical resection of stage I nonsmall cell lung cancer (NSCLC) have been reported to be as high as $28 \%{ }^{1}$ with rates for stage II disease ranging from $20 \%$ to $40 \%{ }^{2,3}$ This suggests that

From the Department of Medicine, ${ }^{a}$ Medical University of South Carolina, Charleston, SC; CALGB Statistical Center, ${ }^{\mathrm{b}}$ Durham, NC; Department of Surgery, ${ }^{\mathrm{c}}$ Duke University Medical Center, Durham, NC; Department of Surgery, ${ }^{\mathrm{d}}$ Brigham and Women's Hospital, Boston, Mass; Department of Surgery, ${ }^{\mathrm{e}}$ Minneapolis Veterans Administration Medical Center, Minneapolis, Minn; Upstate Cancer Center, ${ }^{\mathrm{f}}$ State University of New York Upstate Medical University, New York, NY; and Department of Medicine, ${ }^{\mathrm{g}}$ Masonic Cancer Center, University of Minnesota, Minneapolis, Minn.

Funding: The research for CALGB 159902 was supported in part by National Cancer Institute R21 CA81489-01. It was also supported, in part, by grants from the National Cancer Institute (CA31946) to the Cancer and Leukemia Group B (Monica M. Bertagnolli, MD, Chair) and to the CALGB Statistical Center (Daniel J. Sargent, $\mathrm{PhD}, \mathrm{CA} 33601)$. The content of this manuscript is solely the responsibility of the authors and does not necessarily represent the official views of the National Cancer Institute.

Disclosures: Authors have nothing to disclose with regard to commercial support.

See Appendix 1 for a list of participating institutions.

Received for publication July 12, 2012; revisions received Aug 16, 2012; accepted for publication Aug 23, 2012; available ahead of print Oct 1, 2012.

Address for reprints: Alice M. Boylan, MD, Division of Pulmonary Medicine, Department of Medicine, Medical University of South Carolina, Rm 812 CSB, 96 Jonathan Lucas St, Charleston, SC 29425 (E-mail: boylana@musc.edu).

0022-5223/\$36.00

Copyright (C) 2013 by The American Association for Thoracic Surgery http://dx.doi.org/10.1016/j.jtcvs.2012.08.059 micrometastases are present at the time of resection in a sizable portion of patients. Previous studies have found that cytologic analysis of cells obtained by lavage of the pleural surfaces at the time of surgical resection of NSCLC can identify patients at higher risk for recurrence. $^{4,5}$ A recent meta-analysis of 31 studies from 22 centers found the detection of malignant cells in pleural lavage by cytologic analysis to be an independent predictor of worse NSCLC survival and recommended upstaging such patients by one $\mathrm{T}$ category. ${ }^{5}$ However, the overall rate of positive cytology in this analysis was significantly less than the rate of recurrence, showing that this technique failed to detect many patients in whom recurrent disease would develop.

Cytologic analysis is now recognized as being less sensitive and more subjective than some of the molecular techniques now available to identify genes uniquely expressed by malignant cells. One gene showing particular promise for improving identification of lung cancer cells is telomerase, an enzyme that adds nucleotide repeats to the end of chromosomes, thereby overcoming the normal DNA shortening and cellular senescence. ${ }^{6,7}$ Maintaining telomere length is a critical and perhaps a rate-limiting step in the progression from dysplasia to cancer. ${ }^{6}$ Telomerase is not normally found in somatic human cells and has 


\section{Abbreviations and Acronyms \\ CALGB $=$ Cancer and Leukemia Group B \\ $\mathrm{CI}=$ confidence interval \\ DFS $=$ disease-free survival \\ hTERT $=$ human telomerase reverse transcriptase \\ NSCLC $=$ non-small cell lung cancer \\ OS = overall survival \\ $\mathrm{PCR}=$ polymerase chain reaction \\ RT-PCR $=$ reverse transcriptase polymerase chain reaction}

been reported to be expressed in up to $93 \%$ of all NSCLCs and $100 \%$ of small cell lung cancers. ${ }^{7}$ Furthermore, detection of telomerase in NSCLC tissues has been associated with advanced stage and poorer survival, ${ }^{8}$ and has been shown to improve detection of NSCLC cells in pleural effusions when combined with cytology. ${ }^{9} \mathrm{Be}-$ cause of the association of telomerase with malignant transformation in lung cancer and its association with worse outcome in NSCLC, we hypothesized that identification of cells obtained from pleural lavage that express telomerase would detect patients at risk for recurrent disease.

\section{MATERIALS AND METHODS}

Approval for the study was obtained from the institutional review board for human research at each institution. After informed consent was obtained, patients aged more than 18 years undergoing thoracotomy with curative intent for suspected or confirmed NSCLC were enrolled in Cancer and Leukemia Group B (CALGB) 159902. At the time the chest was opened, the visceral and parietal pleural surfaces were lavaged with 200 $\mathrm{mL}$ of normal saline solution. Pleural lavage liquid was collected in tubes containing ethylenediaminetetraacetic acid and then shipped on ice to a core laboratory for analysis. Total cellular RNA (average yield from each sample $7.8 \pm 3.3 \mu \mathrm{g}$ ) was harvested from the cell pellet and reverse transcribed into cDNA. The cDNA $(2 \mu \mathrm{L})$ was used in hot-start polymerase chain reaction (PCR) with primers based on the human telomerase reverse transcriptase (hTERT) sequence: hTERT (forward, base pair 1790-1811) 5'-AGTGTCTGGAGCAAGTTGCAAA-3' and hTERT (reverse, base pair 1970-1987) 5'-CGTTCTGGCTCCCACGAC-3' (limit of detection: 50 malignant cells/2000 normal cells by spiking cell suspensions with tumor cells). The amplified fragments were separated in $2 \%$ agarose gels and visualized by ethidium bromide staining. As control for RNA integrity and reverse transcription, rRNA levels in each sample were determined by amplifying equal amounts of cDNA $(1 \mu \mathrm{L})$ using the following primers: forward $5^{\prime}$-CTCCGGTCCGTGCCTCCAAG-3' ${ }^{\prime}$ and reverse $5^{\prime}$-CAGAGAATAGCCTGTCTTCAGTC- $3^{\prime}$. Reverse transcriptase polymerase chain reaction (RT-PCR) assays were determined independently as positive or negative by 2 investigators blinded to the clinical and histopathologic information (Figure 1). The limit of detection for this assay was determined to be 50 malignant cells/2000 normal cells by spiking cell suspensions with tumor cells. Telomerase activity was found in the following positive controls: a pancreatic cancer cell line and 2 malignant effusions caused by metastatic adenocarcinoma of the lung. No telomerase activity was found in the following negative controls: cells from a transudative effusion and cells from normal lung. The laboratory lead

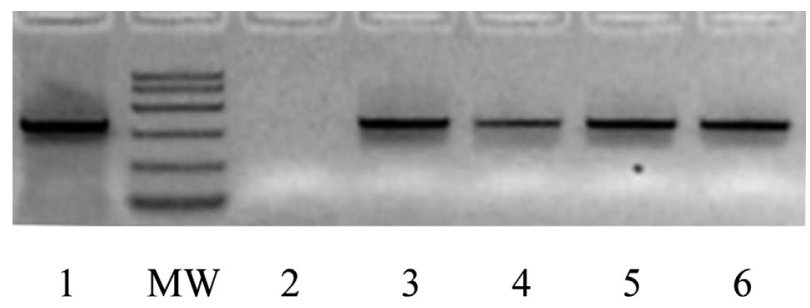

FIGURE 1. Reverse transcriptase polymerase chain reaction (RT-PCR) analysis of human telomerase reverse transcriptase (hTERT) expression in lavage specimens. The messenger RNA levels of hTERT were determined by using RT-PCR. Fragments were resolved on 2\% agarose gels and visualized by ethidium bromide staining. Lane 1 shows expression in the positive control, a lung adenocarcinoma cell line, and lane 2 shows the negative control, normal pleural mesothelial cells. Lanes 4-7 contain pleural lavage specimens. $M W$, Molecular weight markers.

by the first author processed the specimens. Assays were conducted without knowledge of clinical outcomes.

\section{Statistical Methods}

The study was designed to enroll a total of 180 patients with stage I or II NSCLC who underwent complete resection. The sample size was justified with the assumption that $20 \%$ of these patients would have positive test results. With 102 failures observed from the 180 patients, the study has $80 \%$ power at 2 -sided significance level of .05 to detect a 2 -fold increase in the risk of disease recurrence (hazard ratio, 2.0) for marker positives over marker negatives, that is, a decrease of 2-year disease-free survival (DFS) rate from $60 \%$ for marker negatives to $36 \%$ for marker positives. Patient registration and clinical data collection were managed by the CALGB Statistical Center. Clinical data quality was ensured by careful review of data by the CALGB Statistical Center staff and by the study chair. The statistical analyses were performed by CALGB statisticians using SAS 9.1 (SAS Institute Inc, Cary, NC). The association of demographic and clinical variables with telomerase activity (presence or absence of hTERT in cells) was tested using chi-square tests for categoric variables and Wilcoxon rank-sum tests for continuous variables. KaplanMeier curves were used to characterize survival for patients with and without telomerase. The median survival times and the corresponding $95 \%$ confidence intervals (CIs) were computed for overall survival (OS) and DFS. OS was defined as the time from patient registration to death from any cause. DFS was defined as time from patient registration to disease recurrence or death, whichever comes first. The log-rank test was used to compare survival between patient subgroups. Cox proportional hazards model was used to examine the effect of the presence of telomerase on DFS and OS without or with adjustment for other baseline prognostic factors. All $P$ values reported are 2 sided.

\section{RESULTS}

Of the 364 patients accrued (from October 26, 2000, to September 30, 2003), 30 had benign disease, 43 had stage III or IV disease, and 13 had other types of cancer. Samples from 62 patients could not be analyzed because of shipping errors, such as frozen samples, leakage of specimens, loss of shipment, or delays in shipment leading to specimens arriving at room temperature. Two patients withdrew from the study, and the diagnosis was uncertain in 9 patients. Of the patients confirmed to have NSCLC, 194 had stage I/II with adequate samples for analysis (64 presented 
TABLE 1. Patient demographic and baseline clinical characteristics

\begin{tabular}{lccc}
\hline & \multicolumn{2}{c}{ Telomerase activity N (\%) } & \\
\cline { 2 - 3 } \multicolumn{1}{c}{ Characteristics } & $\begin{array}{c}\text { Absent } \\
(\mathbf{N}=\mathbf{1 3 0})\end{array}$ & $\begin{array}{c}\text { Present } \\
(\mathbf{N}=\mathbf{6 4})\end{array}$ & $\begin{array}{c}\text { Total } \\
(\mathbf{N}=\mathbf{1 9 4})\end{array}$ \\
\hline Age median (min, max) & $67(39,86)$ & $70(52,85)$ & $68(39,86)^{*}$ \\
Race & & & \\
$\quad$ White & $123(69.5)$ & $54(30.5)$ & $177 \dagger$ \\
$\quad$ Nonwhite & $5(35.7)$ & $9(64.3)$ & 14 \\
$\quad$ Unknown & $2(66.7)$ & $1(33.3)$ & 3 \\
Gender & & & \\
$\quad$ Male & $67(68.4)$ & $31(31.6)$ & 98 \\
Female & $63(65.6)$ & $33(34.4)$ & 96 \\
ECOG PS & & & \\
$\quad 0$ & $65(64.4)$ & $36(35.6)$ & 101 \\
1, 2 & $58(72.5)$ & $22(27.5)$ & 80 \\
$\quad$ Unknown & $7(53.8)$ & $6(46.2)$ & 13 \\
Histology & & & \\
$\quad$ Adenocarcinoma & $64(68.1)$ & $30(31.9)$ & 94 \\
$\quad$ Other types & $66(66.0)$ & $34(34.0)$ & 100 \\
Stage & & & \\
$\quad$ I & $111(69.4)$ & $49(30.6)$ & 160 \\
II & $19(55.9)$ & $15(44.1)$ & 34 \\
\hline
\end{tabular}

$E C O G$, Eastern Cooperative Oncology Group; $P S$, performance status. *Two-sided $P$ value for age (.082) was from Wilcoxon rank-sum test. $\dagger$ Two-sided $P$ value for race (.016) was from Fisher exact test.

telomerase activity, 79 deaths, and 96 deaths or relapse). On histologic analysis, $48.5 \%$ of cases were adenocarcinomas, $39.7 \%$ were squamous cell carcinomas, $4.1 \%$ were large cell carcinomas, $4.1 \%$ were bronchoalveolar cell carcinomas, and $3.6 \%$ were poorly differentiated carcinomas. The median follow-up time was 60 months (5 years) (range, 17-91 months).

Telomerase activity was not statistically associated with gender, performance status, histology, and stage $(P>.10)$ (Table 1). However, white patients tended to have a higher proportion $(69 \%)$ of telomerase absence than the rest of the patients $(41 \%)$ (Fisher exact test, $P=.016)$, and patients with telomerase absence tended to be younger (median age, 67 years) compared with patients with telomerase present (median age, 70 years) (Wilcoxon rank-sum test, $P=.082$ ). Of 30 samples from patients with benign disease, 22 were tested for telomerase activity, and of these, 4 were found to be positive $(18 \%)$.
OS and DFS were estimated by the Kaplan-Meier method (Table 2 and Figure 2). The estimates of 5-year survival were calculated along with their $95 \%$ CI. The 5-year OS was $62.2 \%(95 \%$ CI, 52.8-70.2) for patients with telomerase absence and 55\% (95\% CI, 40.7-67.1) for patients with telomerase present (log-rank $P=.59$ ). The 5-year DFS was 58.1\% (95\% CI, 48.8-66.2) for patients with telomerase absence and $44.1 \%(95 \% \mathrm{CI}$, 31.0-56.4) for patients with telomerase present (log-rank $P=.23$ ). The Cox univariate model (model 1) and multivariate model (model 2) were fit to assess the effect of telomerase on OS and DFS, respectively. In the multivariate model, covariates of age, race, gender, Eastern Cooperative Oncology Group performance status, histology, and stage were adjusted for. As shown in Table 3, no statistically significant difference in terms of OS and DFS between patients with and without telomerase was present, whereas female patients (performance status $=0$ ) and patients with stage I had more favorable survivals than their counterparts $(P<.06)$ according to the multivariate analysis.

\section{DISCUSSION}

The primary objective of this study was to examine the relationship between telomerase activity (hTERT) in pleural lavage fluids and survival among patients with pathologic stage I and II NSCLC undergoing surgical resection with curative intent. The results suggest that detecting telomerase in cells obtained by lavage of the pleura is not associated with worse DFS or OS in these patients.

Although the specificity of telomerase activity for detecting NSCLC is reportedly high $(85.7 \%$ in bronchial lavage specimens) ${ }^{10}$ the false-positive rate for samples taken from the lungs of patients with suspected NSCLC has been reported to be $10 \%$ to $14.7 \%{ }^{10}$ In all cases, the false-positives were associated with inflammatory processes, perhaps reflecting the presence of activated lymphocytes that have been shown to be capable of expressing telomerase. ${ }^{11}$ Such activated lymphocytes might have been a confounding variable in our study.

The sensitivity and specificity of measument of telomerase activity for detection of malignant cells also vary

TABLE 2. Kaplan-Meier product limit estimates of overall survival and disease-free survival by telomerase activity

\begin{tabular}{|c|c|c|c|c|c|}
\hline Telomerase activity & Patients (n) & Events (n) & Median survival (y) $(95 \%$ CI) & 5-y Survival (\%) $(95 \%$ CI $)$ & Log-rank test $P$ value \\
\hline \multicolumn{6}{|l|}{ OS } \\
\hline Absent & 130 & 50 & NA $(5.6$, NA) & $62.2(52.8-70.2)$ & .59 \\
\hline Present & 64 & 29 & $5.9(4.1, \mathrm{NA})$ & $55.0(40.7-67.1)$ & \\
\hline \multicolumn{6}{|l|}{ DFS } \\
\hline Absent & 130 & 59 & $5.8(4.4, \mathrm{NA})$ & $58.1(48.8-66.2)$ & .23 \\
\hline Present & 64 & 37 & $4.2(2.4,5.9)$ & $44.1(31.0-56.4)$ & \\
\hline
\end{tabular}

$C I$, Confidence interval; $O S$, overall survival; $D F S$, disease-free survival; $N A$, not available. 


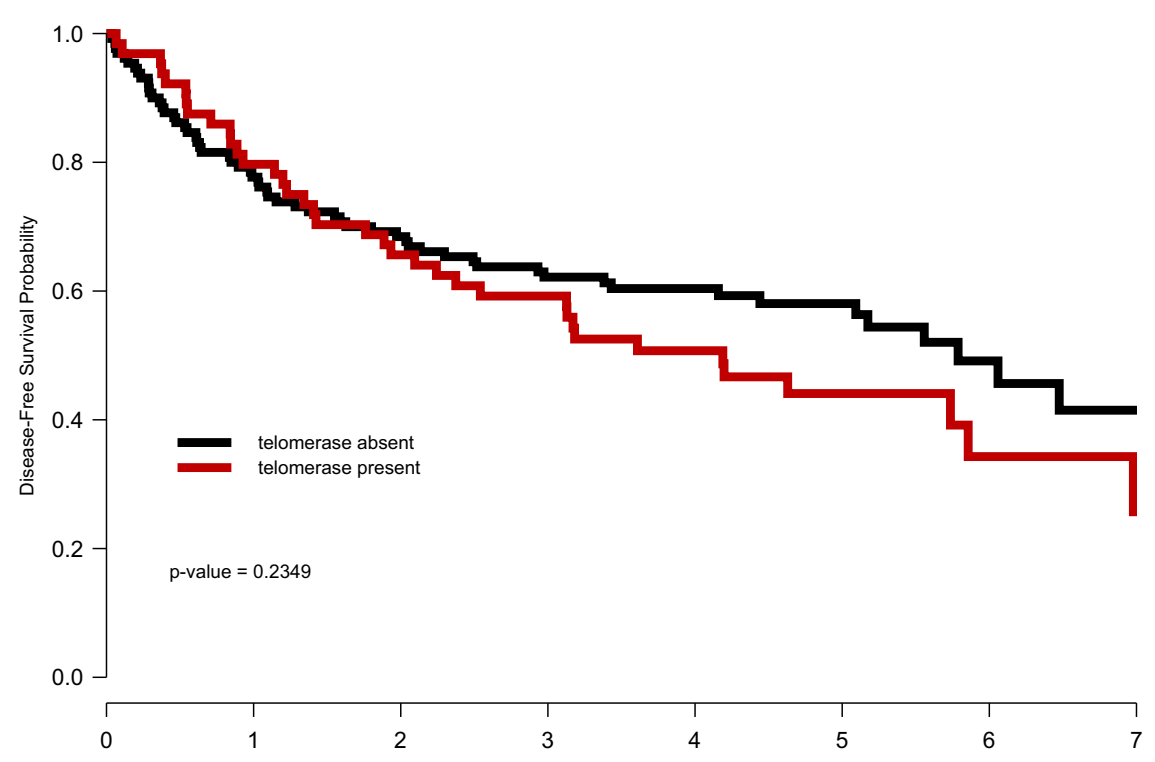

A

Survival Time (years)

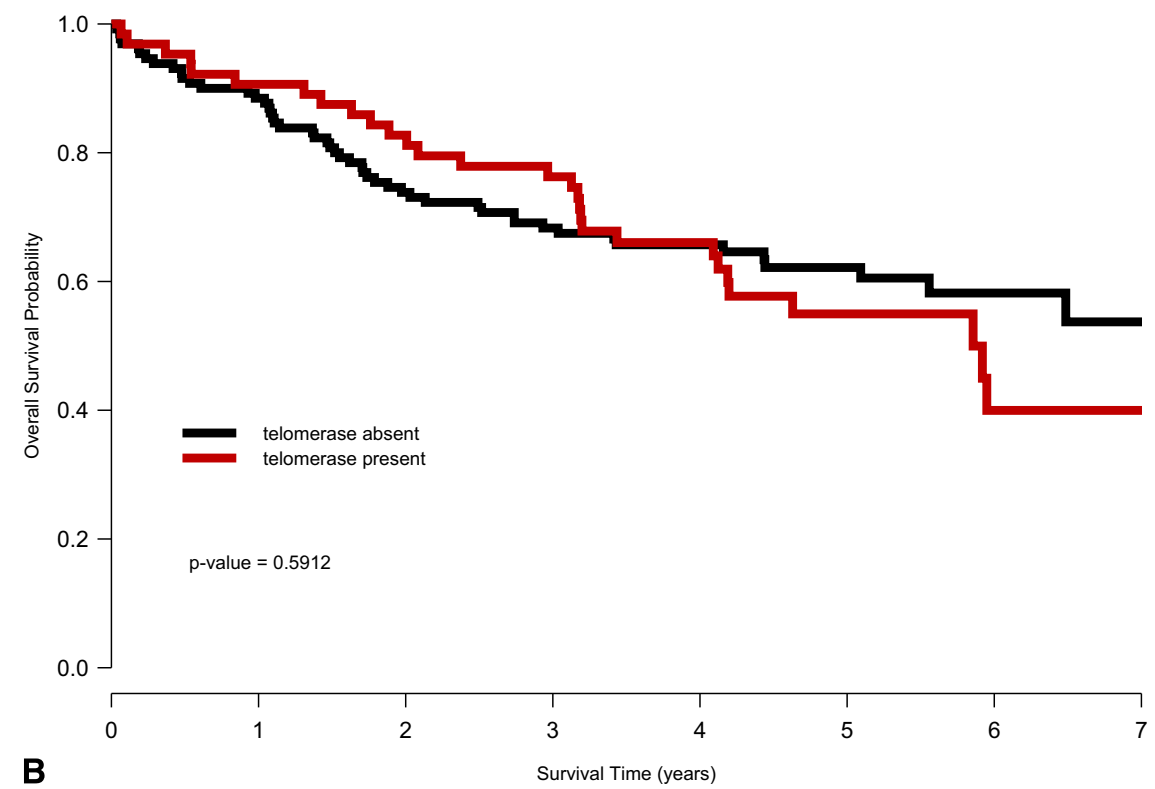

FIGURE 2. Kaplan-Meier curves of (A) disease-free survival and (B) overall survival by telomerase activity.

depending on the technique used, and there is some research to suggest analysis for proteins involved in teleomerase activation is superior to measurements of enzymatic activity. One such protein is hTERT. This protein is a rate-limiting determinant of the enzymatic activity of human telomerase. ${ }^{12}$ Its expression seems to develop early in tumorigenesis ${ }^{13}$ and is associated with shorter survival in NSCLC. ${ }^{14}$ Because of these factors, the ability to use more sensitive PCR technology to detect low numbers of malignant cells and the evidence suggesting that hTERT may be a more specific marker of cancer cells, ${ }^{15}$ we chose to analyze our specimens using RT-PCR to detect expression of hTERT.

Strengths of our study include the fact that it was a prospective, protocol-driven study with multiple centers involved. Patients were prospectively followed for a median of 5 years, and outcome data were correlated with molecular analysis and pathologic stage. Study limitations include a relatively low sample size because of higher than expected numbers of patients with higher stage disease, benign or other cancers, loss of samples due to shipping errors, and a high false-positive rate. Also, RT-PCR was 
TABLE 3. Relationship between telomerase activity and overall survival/disease-free survival from Cox proportional hazard model without (model 1) and with (model 2) adjustments for other prognostic factors

\begin{tabular}{|c|c|c|c|c|c|c|}
\hline & \multicolumn{3}{|c|}{ Overall survival } & \multicolumn{3}{|c|}{ Disease-free survival } \\
\hline & HR & $95 \% \mathrm{CI}$ & $P$ value & HR & $\mathbf{9 5} \% \mathrm{CI}$ & $P$ value \\
\hline \multicolumn{7}{|l|}{ Model $1 *$} \\
\hline Telomerase present vs absent & 1.13 & $0.72-1.79$ & .59 & 1.28 & $0.85-1.94$ & .24 \\
\hline \multicolumn{7}{|l|}{ Model $2 \dagger$} \\
\hline Telomerase present vs absent & 1.06 & $0.64-1.76$ & .82 & 1.26 & $0.80-1.98$ & .32 \\
\hline Age (change by $1-y)$ & 1.02 & $0.99-1.05$ & .24 & 1.02 & $0.99-1.04$ & .24 \\
\hline White vs nonwhite & 0.77 & $0.34-1.74$ & .53 & 0.74 & $0.35-1.56$ & .43 \\
\hline Male vs female & 1.91 & $1.17-3.14$ & .01 & 1.71 & $1.10-2.65$ & .018 \\
\hline $\mathrm{PS}=0 \mathrm{vs} 1 / 2$ & 0.60 & $0.37-0.97$ & .037 & 0.65 & $0.42-1.02$ & .059 \\
\hline Adeno vs others & 1.06 & $0.65-1.72$ & .82 & 1.23 & $0.79-1.90$ & .35 \\
\hline Stage I vs II & 0.54 & $0.32-0.92$ & .022 & 0.57 & $0.35-0.94$ & .027 \\
\hline
\end{tabular}

$H R$, Hazard ratio; $C I$, confidence interval; $P S$, performance status; Adeno, adenocarcinoma. *Univariate model without any covariates. $\dagger$ Multivariate model adjusting for age, race, gender, Eastern Cooperative Oncology Group performance status, histology type, and stage.

used, which is less sensitive than currently available quantitative PCR.

\section{CONCLUSIONS}

The use of a PCR-based method to detect telomerase gene expression in cells obtained by pleural lavage of patients with stage I or II NSCLC failed to identify patients at risk for recurrence. It may be that the number of malignant cells in the pleural space is too few for even a sensitive method of detection such as PCR. Given the high false-positive rate in benign inflammatory lesions, it may also be that differentiation of patients with higher risk of recurrence requires the association of telomerase activity with the presence of reactive lymphocytes or other molecular markers of malignancy to improve prognostic differentiation.

\section{References}

1. Lardinois D, Suter H, Hakki H, Rousson V, Betticher D, Ris HB. Morbidity, survival, and site of recurrence after mediastinal lymph-node dissection versus systematic sampling after complete resection for non-small cell lung cancer. Ann Thorac Surg. 2005;80:268-75.

2. Feng QF, Wang M, Wang LJ, Yang ZY, Zhang YG, Zhang DW, et al. A study of postoperative radiotherapy in patients with non-small-cell lung cancer: a randomized trial. Int J Radiat Oncol Biol Phys. 2000;47:925-9.

3. Luzzi L, Paladini P, Ghiribelli C, Voltolini L, Di Bisceglie M, D’Agata A, et al. Assessing the prognostic value of the extent of mediastinal lymph node infiltration in surgically-treated non-small cell lung cancer (NSCLC). Lung Cancer. 2000;30:99-105.

4. Nakagawa T, Okumura N, Kokado Y, Miyoshi K, Matsuoka T, Kameyama K. Clinical relevance of intraoperative pleural lavage cytology in non-small cell lung cancer. Ann Thorac Surg. 2007;83:204-8.

5. Lim E, Clough R, Goldstraw P, Edmonds L, Aokage K, Yoshida J, et al. Impact of positive pleural lavage cytology on survival in patients having lung resection for non-small-cell lung cancer: an international individual patient data meta-analysis. J Thorac Cardiovasc Surg. 2009;139:1441-6.

6. Fernandez-Garcia I, Ortiz-de-Solorzano C, Montuenga LM. Telomeres and telomerase in lung cancer. J Thorac Oncol. 2008;3:1085-8.

7. Kumaki F, Kawai T, Hiroi S, Shinomiya N, Ozeki Y, Ferrans VJ, et al. Telomerase activity and expression of human telomerase RNA component and human telomerase reverse transcriptase in lung carcinomas. Hum Pathol. 2001;32: 188-95.

8. Albanell J, Lonardo F, Rusch V, Engelhardt M, Langenfeld J, Han W, et al. High telomerase activity in primary lung cancers: association with increased cell proliferation rates and advanced pathologic stage. J Natl Cancer Inst. 1997;89: 1609-15.
9. Dikmen G, Dikmen E, Kara M, Sahin E, Dogan P, Ozdemir N. Diagnostic implications of telomerase activity in pleural effusions. Eur Respir J. 2003;22: 422-6.

10. Dikmen E, Kara M, Dikmen G, Cakmak H, Dogan P. Detection of telomerase activity in bronchial lavage as an adjunct to cytological diagnosis in lung cancer. Eur J Cardiothorac Surg. 2003;23:194-9; discussion 199-20.

11. Hiyama K, Hirai Y, Kyoizumi S, Akiyama M, Hiyama E, Piatyszek MA, et al. Activation of telomerase in human lymphocytes and hematopoietic progenitor cells. J Immunol. 1995; 155:3711-5.

12. Poole JC, Andrews LG, Tollefsbol TO. Activity, function, and gene regulation of the catalytic subunit of telomerase (hTERT). Gene. 2001;269:1-12.

13. Kolquist KA, Ellisen LW, Counter CM, Meyerson M, Tan LK, Weinberg RA, et al. Expression of TERT in early premalignant lesions and a subset of cells in normal tissues. Nat Genet. 1998;19:182-6.

14. Marchetti A, Pellegrini C, Buttitta F, Falleni M, Romagnoli S, Felicioni L, et al. Prediction of survival in stage I lung carcinoma patients by telomerase function evaluation. Lab Invest. 2002;82:729-36.

15. Mu J, Wei LX. Telomere and telomerase in oncology. Cell Res. 2002;12:1-7.

\section{APPENDIX 1. PARTICIPATING INSTITUTIONS}

Department of Medicine, Medical University of South Carolina, Charleston, SC; supported by CA03927.

CALGB Statistical Center, Duke University Medical Center, Durham, NC; supported by CA33601.

Department of Surgery, Duke University Medical Center,

Durham, NC; supported by CA47577.

Department of Surgery, Brigham and Women's Hospital,

Boston, Mass; supported by CA32291.

Department of Surgery, Minneapolis Veterans Administration Medical Center; Minneapolis, Minn; supported by CA16450.

Upstate Cancer Center, State University of New York Upstate Medical University, Syracuse, NY; supported by CA21060.

Department of Medicine, University of Minnesota, Minneapolis, Minn; supported by CA16450.

Dana-Farber Cancer Institute, Boston, Mass, Harold J Burstein, MD, PhD; supported by CA32291.

Duke University Medical Center, Durham, NC, Jeffrey Crawford, MD; supported by CA47577.

Medical University of South Carolina, Charleston, SC, Mark Green, MD; supported by CA03927. 
Southeast Cancer Control Consortium Inc. CCOP, Goldsboro, NC, James N. Atkins, MD; supported by CA45808.

State University of New York Upstate Medical University, Syracuse, NY, Stephen L. Graziano, MD; supported by CA2 21060 .

University of Maryland Greenebaum Cancer Center, Baltimore, Md, Martin Edelman, MD; supported by CA31983.
University of Minnesota, Minneapolis, Minn, Bruce A Peterson, MD; supported by CA16450.

University of Missouri/Ellis Fischel Cancer Center, Columbia, Mo, Michael C. Perry, MD; supported by CA12046.

University of Nebraska Medical Center, Omaha, Neb, Anne Kessinger, MD; supported by CA77298. 